FACTA UNIVERSITATIS (NIŠ)

Ser. Math. Inform. Vol. 32, No 1 (2017), 59-72

DOI:10.22190/FUMI1701059A

\title{
ON SOME SUBSETS OF SOFT WEAK STRUCTURES
}

\begin{abstract}
Alaa M. Abd El-latif
Abstract. The main purpose of this paper, is to list the main properties which give the deviations between soft weak structure [17] and that in soft topological spaces [15] and supra soft topological spaces [7], which is supported by counter examples. Moreover, we introduce and study the basic properties of the soft structures $\pi(s w o), \sigma(s w o), \alpha(s w o)$, $\beta($ swo $)$ and $\rho(s w o)$.

Keywords: Soft weak structure, Soft generalized topological spaces, Soft minimal spaces, $\pi(s w o), \sigma(s w o), \alpha(s w o), \beta(s w o)$ and $\rho(s w o)$.
\end{abstract}

\section{Introduction}

Csásázr [3] introduced a generalized structure called generalized topology. Also, Csásázr [2,4], introduced and studied generalized operators. After then, Csásázr [5] has introduced a new notion of structures called weak structure (briefly, $W S$ ). So that, every generalized topology [3] and every minimal structure [13] is a WS. In 2007, Arpad Szaz [1] succeed to introduce an application on the minimal spaces and generalized spaces. In [5], Csásázr defined some structures and operators under more general conditions, which are investigated in detail in $[6,8,16]$.

Molodtsov [14] initiated the concept of soft set theory as a new mathematical tool for dealing with uncertainties. In 2011, Shabir et al. [15] initiated the study of soft topological spaces. The notions of supra soft topological space were first introduced by El-Sheikh et al. [7]. The notion of soft generalized topological space was introduced by Jyothis et al. in [10], which is generalized in [9] to notion of soft minimal spaces. In 2016, Zakari et al. [17] introduced the concepts of soft weak structure $\mathcal{S W}$ over a universe $X$ with a fixed set of parameters $E$.

Our aims of this paper, is to study the deviations between soft weak structure and that in soft topological spaces and supra soft topological spaces. New soft structures, as a generalizations to thats in $[5,7,11,6]$ are introduced and their properties are investigated

Received July 29, 2016; accepted December 12, 2016

2010 Mathematics Subject Classification. Primary 54A40, 54C08; Secondary 06D72 


\section{Preliminaries}

In this section, we present the basic definitions and results of soft set theory which will be needed in the sequel. For more detail see $[7,15,17,18]$

Definition 2.1. [14] Let $X$ be an initial universe and $E$ be a set of parameters. Let $P(X)$ denote the power set of $X$ and $A$ be a non-empty subset of $E$. A pair $(F, A)$ denoted by $F_{A}$ is called a soft set over $X$, where $F$ is a mapping given by $F: A \rightarrow P(X)$. In other words, a soft set over $X$ is a parametrized family of subsets of the universe $X$. For a particular $e \in A, F(e)$ may be considered the set of $e$-approximate elements of the soft set $(F, A)$ and if $e \notin A$, then $F(e)=\varphi$ i.e $F_{A}=\{F(e): e \in A \subseteq E, F: A \rightarrow P(X)\}$. The family of all these soft sets denoted by $S S(X)_{A}$.

Definition 2.2. [15] Let $(F, E)$ be a soft set over $X$ and $x \in X$. We say that $x \in(F, E)$ read as $x$ belongs to the soft set $(F, E)$ whenever $x \in F(e)$ for all $e \in E$.

Definition 2.3. [15] Let $\tau$ be a collection of soft sets over a universe $X$ with a fixed set of parameters $E$, then $\tau \subseteq S S(X)_{E}$ is called a soft topology on $X$ if

1. $\tilde{X}, \tilde{\varphi} \in \tau$, where $\tilde{\varphi}(e)=\varphi$ and $\tilde{X}(e)=X, \forall e \in E$,

2. the union of any number of soft sets in $\tau$ belongs to $\tau$,

3. the intersection of any two soft sets in $\tau$ belongs to $\tau$.

The triplet $(X, \tau, E)$ is called a soft topological space over $X$.

Definition 2.4. [18] The soft set $(F, E) \in S S(X)_{E}$ is called a soft point in $X_{E}$ if there exist $x \in X$ and $e \in E$ such that $F(e)=\{x\}$ and $F\left(e^{\prime}\right)=\varphi$ for each $e^{\prime} \in E-\{e\}$, and the soft point $(F, E)$ is denoted by $x_{e}$.

Definition 2.5. [18] The soft point $x_{e}$ is said to be belonging to the soft set $(G, E)$, denoted by $x_{e} \tilde{\in}(G, E)$, if for the element $e \in E, x \in G(e)$.

Definition 2.6. [9] Let $\tilde{m}$ be a collection of soft sets over a universe $X$ with a fixed set of parameters $E$, then $\tilde{m}$ is called a soft minimal space if and only if $\tilde{X}, \tilde{\varphi} \in \tilde{m}$.

Definition 2.7. [17] Let $\mathcal{S W}$ be a collection of soft sets over a universe $X$ with a fixed set of parameters $E$, then $\mathcal{S W}$ is called a soft weak structure if and only if $\tilde{\varphi} \in \mathcal{S W}$. The members of $\mathcal{S W}$ are said to be $\mathcal{S} \mathcal{W}$-open soft sets in $X$. A soft set $(F, E)$ over $X$ is said to be a $\mathcal{S W}$-closed soft set, if its relative complement $(F, E)^{\tilde{c}}$ is $\mathcal{S} \mathcal{W}$-open soft set. We denote the set of all $\mathcal{S} \mathcal{W}$-open soft sets over $X$ by $\operatorname{swo}(X)$ and the set of all $\mathcal{S} \mathcal{W}$-closed soft sets by $\operatorname{swc}(X)$. 
Definition 2.8. [17] Let $\mathcal{S W}$ be a soft weak structure over $X$ with a fixed set of parameters $E$. Then, the $\mathcal{S} \mathcal{W}$-soft interior of $(G, E)$, denoted by $i_{s w}(G, E)$ is the soft union of all $\mathcal{S W}$-open soft subsets of $(G, E)$ i.e

$i_{s w}(G, E)=\tilde{\cup}\{(H, E):(H, E)$ is $\mathcal{S W}$ - open soft set and $(H, E) \tilde{\subseteq}(G, E)\}$.

Definition 2.9. [17] Let $\mathcal{S W}$ be a soft weak structure over $X$ with a fixed set of parameters $E$. Then, the $\mathcal{S} \mathcal{W}$-soft closure of $(F, E)$, denoted by $c_{s w}(F, E)$ is the soft intersection of all $\mathcal{S} \mathcal{W}$-closed soft supersets of $(F, E)$ i.e

$c_{s w}(F, E)=\tilde{\cap}\{(H, E):(H, E)$ is $\mathcal{S W}$ - closed soft set and $(F, E) \tilde{\subseteq}(H, E)\}$.

Theorem 2.1. [17] Let $\mathcal{S W}$ be a soft weak structure over $X$ with a fixed set of parameters $E$ and $(F, E),(G, E) \in S S(X)_{E}$, then:

$$
\begin{aligned}
& \text { 1. } i_{s w}(F, E) \tilde{\subseteq}(F, E) \tilde{\subseteq} c_{s w}(F, E) \text {. } \\
& \text { 2. } c_{s w}\left(c_{s w}(F, E)\right)=c_{s w}(F, E) \text { and } i_{s w}\left(i_{s w}(F, E)\right)=i_{s w}(F, E) \text {. } \\
& \text { 3. If }(F, E) \tilde{\subseteq}(G, E) \text {, then } i_{s w}(F, E) \tilde{\subseteq} i_{s w}(G, E) \text { and } c_{s w}(F, E) \tilde{\subseteq} c_{s w}(G, E) \text {. } \\
& \text { 4. } c_{s w}(\tilde{X}-(F, E))=\tilde{X}-i_{s w}(F, E) \text { and } i_{s w}(\tilde{X}-(F, E))=\tilde{X}-c_{s w}(F, E) .
\end{aligned}
$$

Definition 2.10. [17] Let $\mathcal{S W}$ be a soft weak structure over $X$ with a fixed set of parameters $E$ and $(F, \underset{\sim}{E}) \in S S(X)_{E}$. Then, $(F, E)$ is called $\mathcal{S} \mathcal{W}$-soft dense set if and only if $c_{s w}(F, E)=\tilde{X}$.

\section{On Soft Weak Structure}

In this section, we list the main properties which give the deviations between soft weak structure [17] and that in soft topological spaces [15] and supra soft topological spaces [7], which is supported by a counter example.

Theorem 3.1. Let $\mathcal{S W}$ be a soft weak structure over $X$ with a fixed set of parameters $E$ and $(F, E) \in S S(X)_{E}$, then:

1. If $(F, E)$ is $\mathcal{S W}$-open soft set, then $(F, E)=i_{\text {sw }}(F, E)$.

2. If $(F, E)$ is $\mathcal{S} \mathcal{W}$-closed soft set, then $(F, E)=c_{s w}(F, E)$.

Proof. 1. Let $(F, E) \in \operatorname{swo}(X)$, then $(F, E) \tilde{\simeq} \tilde{\cup}\{(H, E):(H, E) \in \operatorname{swo}(X)$ and $(H, E) \tilde{\simeq}(F, E)\}=i_{s w}(F, E)$. But, $i_{s w}(F, E) \tilde{\simeq}(F, E)$ from Theorem 2.1 (1) and thus $(F, E)=i_{s w}(F, E)$.

2. By a similar way. 
Remark 3.1. The converse of Theorem 3.1 is not true in general as shown in the following example.

Example 3.1. Suppose that there are four alternatives in the universe of houses $X=$ $\left\{h_{1}, h_{2}, h_{3}, h_{4}\right\}$ and consider $E=\left\{e_{1}, e_{2}\right\}$ be the set of decision parameters which stand for "wooden" and "green surroundings" respectively. Let $\mathcal{S W}=\left\{\tilde{\varphi},\left(F_{1}, E\right),\left(F_{2}, E\right),\left(F_{3}, E\right)\right\}$, where $\left(F_{1}, E\right),\left(F_{2}, E\right),\left(F_{3}, E\right)$ are three soft sets over $X$ representing the attractiveness of the houses which Mr. $A$ and Mr. $B$ are going to buy defined as follows:

$F_{1}\left(e_{1}\right)=\left\{h_{1}\right\}, \quad F_{1}\left(e_{2}\right)=\left\{h_{2}, h_{3}\right\}$,

$F_{2}\left(e_{1}\right)=\left\{h_{2}\right\}, \quad F_{2}\left(e_{2}\right)=\left\{h_{4}\right\}$,

$F_{3}\left(e_{1}\right)=\left\{h_{2}\right\}, \quad F_{3}\left(e_{2}\right)=\left\{h_{3}\right\}$.

For the soft set $(G, E)$, where:

$G\left(e_{1}\right)=\left\{h_{1}, h_{2}\right\}, \quad G\left(e_{2}\right)=\left\{h_{2}, h_{3}, h_{4}\right\}$,

we have $i_{s w}(G, E)=(G, E)$, but $(G, E) \notin s w o(X)$.

On the other hand, the soft set $(H, E)$, where:

$H\left(e_{1}\right)=\left\{h_{3}, h_{4}\right\}, \quad H\left(e_{2}\right)=\left\{h_{1}, h_{4}\right\}$,

we have $c_{s w}(H, E)=(H, E)$, but $(H, E) \notin \operatorname{swc}(X)$.

The following results were introduced before in [17], but by using the crisp point $x \in X$. So, we reintroduce it again by using the soft point $x_{e} \tilde{\in} \tilde{X}$, which is more general.

Theorem 3.2. Let $\mathcal{S W}$ be a soft weak structure over $X$ with a fixed set of parameters $E$ and $(F, E),(G, E) \in S S(X)_{E}$, then:

1. $x_{e} \tilde{\epsilon} c_{s w}(F, E)$ if and only if $(G, E) \tilde{\cap}(F, E) \neq \tilde{\varphi}$ for all $\mathcal{S W}$-open soft set $(G, E)$ and $x_{e} \tilde{\in}(G, E)$.

2. $x_{e} \tilde{\in} i_{s w}(F, E)$ if and only if there exists a $\mathcal{S W}$-open soft set $(G, E)$ such that $x_{e} \tilde{\in}(G, E) \tilde{\subseteq}(F, E)$.

Proof. 1. Suppose that there exists a $\mathcal{S W}$-open soft set $(G, E)$ such that $(G, E) \tilde{\cap}(F, E)=\tilde{\varphi}$ and $x_{e} \tilde{\in}(G, E)$. Then, $(F, E) \tilde{\subseteq}(G, E)^{\tilde{c}}$, and therefore $x_{e} \tilde{\notin} c_{s w}(F, E)$. On the other hand, if $x_{e} \tilde{\notin} c_{s w}(F, E)$, then there exists a $\mathcal{S} \mathcal{W}$ closed soft set $(G, E)$ with $(F, E) \tilde{\subseteq}(G, E)$ and $x_{e} \tilde{\notin}(G, E)$. Hence, $x_{e} \tilde{\in}(G, E)^{\tilde{c}} \in$ $\operatorname{swo}(X)$ and $(G, E)^{\tilde{c}} \tilde{\cap}(F, E)=\tilde{\varphi}$.

2. Follows easily from Definition 2.8.

Definition 3.1. Let $\mathcal{S W}$ be a soft weak structure over $X$ with a fixed set of parameters $E$ and $(G, E) \in S S(X)_{E}$. Then, $x_{e} \tilde{\in} \tilde{X}$ is called $\mathcal{S} \mathcal{W}$-limit soft point of $(G, E)$ if $\left((G, E)-x_{e}\right) \tilde{\cap}(H, E) \neq \tilde{\varphi} \forall(H, E) \in \operatorname{swo}(X)$ and $(H, E)$ containing $x_{e}$. The set of all $\mathcal{S W}$-limit soft points of $(G, E)$ is called the $\mathcal{S W}$-soft derived of $(G, E)$ and denoted by $d_{s w}(G, E)$.

Theorem 3.3. Let $\mathcal{S W}$ be a soft weak structure over $X$ with a fixed set of parameters $E$ and $(F, E),(G, E) \in S S(X)_{E}$, then: 
1. If $(F, E) \tilde{\subseteq}(G, E)$, then $d_{s w}(F, E) \tilde{\subseteq} d_{s w}(G, E)$.

2. $d_{s w}(F, E) \tilde{\simeq} c_{s w}(F, E)$.

3. If $(F, E) \in \operatorname{swc}(X)$, then $d_{s w}(F, E) \tilde{\simeq}(F, E)$.

4. $c_{s w}(F, E)=(F, E) \cup \tilde{U} d_{s w}(F, E)$.

Proof. 1. Assume that $x_{e} \tilde{\notin} d_{s w}(G, E)$. Then, there exists $(H, E) \in \operatorname{swo}(X)$ containing $x_{e}$ such that $\left((G, E)-x_{e}\right) \tilde{\cap}(H, E)=\tilde{\varphi}$. Since $(F, E) \tilde{\subseteq}(G, E)$, $\left((F, E)-x_{e}\right) \tilde{\cap}(H, E)=\tilde{\varphi}$. this means that, $x_{e} \tilde{\notin} d_{s w}(F, E)$.

2. Assume that $x_{e} \tilde{\notin} c_{s w}(F, E)$. Then, there exists $(H, E) \in \operatorname{swo}(X)$ containing $x_{e}$ such that $(F, E) \tilde{\cap}(H, E)=\tilde{\varphi}$ from Theorem $3.2(1)$. It follows, $((F, E)-$ $\left.x_{e}\right) \tilde{\cap}(H, E)=\tilde{\varphi}$. Thus, $x_{e} \ddot{\notin} d_{s w}(F, E)$.

3. Suppose that $(F, E) \in \operatorname{swc}(X)$ and $x_{e} \tilde{\notin}(F, E)$. Then $x_{e} \tilde{\epsilon}(F, E)^{\tilde{c}}$ and $(F, E)^{\tilde{c}} \in \operatorname{swo}(X)$. Therefore, $\left((F, E)-x_{e}\right) \tilde{\cap}(F, E)^{\tilde{c}}=\tilde{\varphi}$. This means that $x_{e} \tilde{\notin} d_{s w}(F, E)$.

4. It is clear that $c_{s w}(F, E) \supseteq(F, E) \tilde{\cup} d_{s w}(F, E)$ from (2). For the other inclusion, assume that $x_{e} \tilde{\notin}(F, E) \tilde{\cup} d_{s w}(F, E)$, and hence $x_{e} \ddot{\notin}(F, E)$ and $x_{e} \tilde{\notin} d_{s w}(F, E)$. Hence, there exists $(G, E) \in \operatorname{swo}(X)$ containing $x_{e}$ which satisfies the property $\left((F, E)-x_{e}\right) \tilde{\cap}(G, E)=\tilde{\varphi}$, and so $(F, E) \tilde{\cap}(G, E)=\tilde{\varphi}$, where $x_{e} \tilde{\notin}(F, E)$. Therefore, $x_{e} \tilde{\notin} c_{s w}(F, E)$. This completes the proof.

In the next theorem, we list the main properties of the operations which give the deviations between these operations in soft weak structures and that in soft topological spaces and supra soft topological spaces.

Theorem 3.4. Let $\mathcal{S W}$ be a soft weak structure over $X$ with a fixed set of parameters $E$ and $(F, E),(G, E) \in S S(X)_{E}$. Then:

$$
\begin{aligned}
& \text { 1. } i_{s w}((F, E) \tilde{\cap}(G, E)) \tilde{\subseteq} i_{s w}(F, E) \tilde{\cap} i_{s w}(G, E) . \\
& \text { 2. } c_{s w}(F, E) \tilde{\cup} c_{s w}(G, E) \tilde{\subseteq} c_{s w}((F, E) \tilde{\cup}(G, E)) . \\
& \text { 3. } d_{s w}(F, E) \tilde{\cup} d_{s w}(G, E) \tilde{\subseteq} d_{s w}((F, E) \tilde{\cup}(G, E)) .
\end{aligned}
$$

Proof. 1. Since $(F, E) \tilde{\cap}(G, E) \tilde{\subseteq}(F, E)$ and

$$
(F, E) \tilde{\cap}(G, E) \tilde{\subseteq}(G, E), i_{s w}((F, E) \tilde{\cap}(G, E)) \tilde{\subseteq} i_{s w}(F, E)
$$

and $i_{s w}((F, E) \tilde{\cap}(G, E)) \tilde{\subseteq} i_{s w}(G, E)$ from Theorem 2.1 (3). Hence,

$$
i_{s w}((F, E) \tilde{\cap}(G, E)) \tilde{\subseteq} i_{s w}(F, E) \tilde{\cap} i_{s w}(G, E) .
$$


2. Since

$$
(F, E),(G, E) \tilde{\subseteq}(F, E) \tilde{\cup}(G, E), c_{s w}(F, E) \tilde{\subseteq} c_{s w}((F, E) \tilde{\cup}(G, E))
$$

and $\left.c_{s w}(G, E) \tilde{\simeq} c_{s w}((F, E)) \tilde{\cup}(G, E)\right)$ from Theorem 2.1 (3), it follows that $c_{s w}(F, E) \tilde{\cup} c_{s w}(G, E) \tilde{\subseteq} c_{s w}((F, E) \tilde{\cup}(G, E))$.

3. By a similar argument.

Remark 3.2. The converse of Theorem 3.4 is not true in general as shown in the following examples.

Examples 3.1. 1. Suppose that there are four alternatives in the universe of cars $X=\left\{c_{1}, c_{2}, c_{3}, c_{4}\right\}$ and consider $E=\left\{e_{1}, e_{2}\right\}$ be the set of decision parameters which stand for "expensive" and "color" respectively. Let $\mathcal{S W}=$ $\left\{\tilde{\varphi},\left(F_{1}, E\right),\left(F_{2}, E\right),\left(F_{3}, E\right),\left(F_{4}, E\right)\right\}$, where $\left(F_{1}, E\right),\left(F_{2}, E\right),\left(F_{3}, E\right),\left(F_{4}, E\right)$ are four soft sets over $X$ representing the attractiveness of the cars which Mr. $V$ and Mr. $U$ are going to buy defined as follows:

$F_{1}\left(e_{1}\right)=\left\{c_{1}, c_{3}\right\}, \quad F_{1}\left(e_{2}\right)=\left\{c_{1}, c_{4}\right\}$,

$F_{2}\left(e_{1}\right)=\left\{c_{1}, c_{2}\right\}, \quad F_{2}\left(e_{2}\right)=\left\{c_{1}, c_{3}\right\}$,

$F_{3}\left(e_{1}\right)=\left\{c_{2}, c_{4}\right\}, \quad F_{3}\left(e_{2}\right)=\left\{c_{2}, c_{3}\right\}$,

$F_{4}\left(e_{1}\right)=\left\{c_{1}, c_{2}, c_{4}\right\}, \quad F_{4}\left(e_{2}\right)=\left\{c_{1}, c_{2}, c_{3}\right\}$.

For the soft sets $\left(F_{1}, E\right)$ and $\left(F_{2}, E\right)$ we have: $i_{s w}\left(F_{1}, E\right)=\left(F_{1}, E\right)$ and $i_{s w}\left(F_{2}, E\right)=\left(F_{2}, E\right)$, so $i_{s w}\left(F_{1}, E\right) \tilde{\cap} i_{s w}\left(F_{2}, E\right)=(W, E)$, where

$W\left(e_{1}\right)=\left\{c_{1}\right\}, \quad W\left(e_{2}\right)=\left\{c_{1}\right\}$. Also, $i_{s w}\left(\left(F_{1}, E\right) \tilde{\cap}\left(F_{2}, E\right)\right)=\tilde{\varphi}$. Thus, $i_{s w}\left(\left(F_{1}, E\right) \tilde{\cap}\left(F_{2}, E\right)\right) \nsupseteq i_{s w}\left(F_{1}, E\right) \tilde{\cap} i_{s w}\left(F_{2}, E\right)$.

2. Suppose that there are four alternatives in the universe of cameras $X=$ $\left\{c_{1}, c_{2}, c_{3}, c_{4}\right\}$ and consider $E=\left\{e_{1}, e_{2}\right\}$ be the set of decision parameters which stand for "size" and "pixels" respectively. Let $\mathcal{S W}=\left\{\tilde{\varphi},\left(F_{1}, E\right),\left(F_{2}, E\right),\left(F_{3}, E\right),\left(F_{4}, E\right),\left(F_{5}, E\right)\right\}$, where $\left(F_{1}, E\right),\left(F_{2}, E\right),\left(F_{3}, E\right),\left(F_{4}, E\right),\left(F_{5}, E\right)$ are five soft sets over $X$ representing the attractiveness of the cameras which Mr. $M$ and Mr. $N$ are going to buy defined as follows:

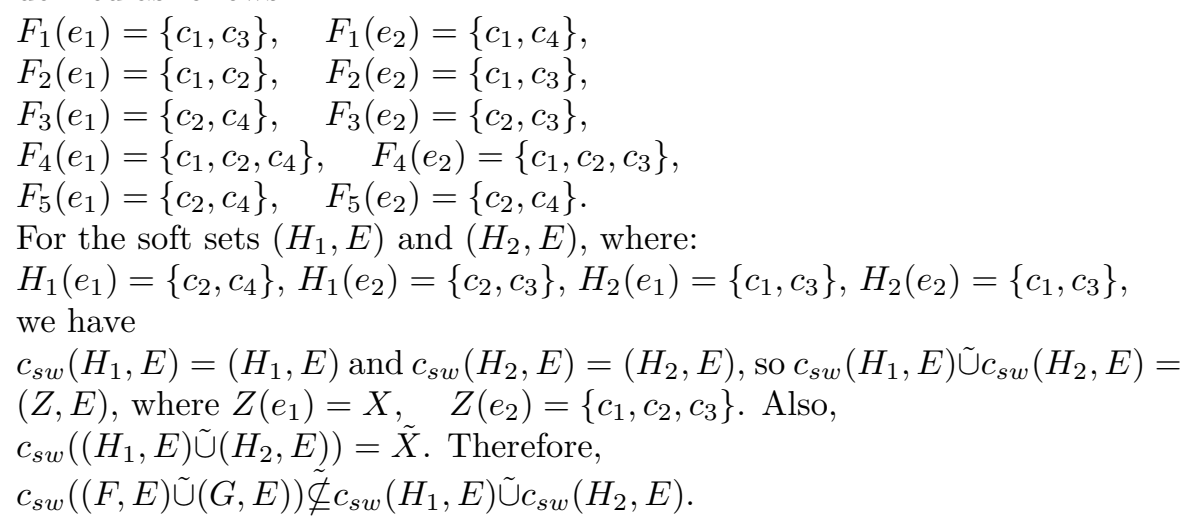


3. Suppose that there are four alternatives in the universe of houses $X=\left\{h_{1}, h_{2}, h_{3}, h_{4}\right\}$ and consider $E=\left\{e_{1}, e_{2}\right\}$ be the set of decision parameters which stand for "expensive" and "position" respectively. Let $\mathcal{S} \mathcal{W}=\left\{\tilde{\varphi},\left(F_{1}, E\right),\left(F_{2}, E\right),\left(F_{3}, E\right),\left(F_{4}, E\right),\left(F_{5}, E\right)\right\}$, where

$\left(F_{1}, E\right),\left(F_{2}, E\right),\left(F_{3}, E\right),\left(F_{4}, E\right),\left(F_{5}, E\right)$ are five soft sets over $X$ representing the attractiveness of the houses which Mr. $A$ and Mr. $B$ are going to buy defined as follows:

$$
\begin{aligned}
& F_{1}\left(e_{1}\right)=\left\{h_{1}, h_{2}\right\}, \quad F_{1}\left(e_{2}\right)=\left\{h_{2}, h_{4}\right\}, \\
& F_{2}\left(e_{1}\right)=\left\{h_{2}, h_{4}\right\}, \quad F_{2}\left(e_{2}\right)=\left\{h_{1}, h_{3}\right\} \\
& F_{3}\left(e_{1}\right)=\left\{h_{1}, h_{3}, h_{4}\right\}, \quad F_{3}\left(e_{2}\right)=\left\{h_{1}, h_{3}, h_{4}\right\}, \\
& F_{4}\left(e_{1}\right)=X, \quad F_{4}\left(e_{2}\right)=\left\{h_{1}, h_{3}\right\}, \\
& F_{5}\left(e_{1}\right)=\left\{h_{1}, h_{3}, h_{4}\right\}, \quad F_{5}\left(e_{2}\right)=X . \\
& \text { Let }(G, E) \text { and }(H, E) \text { be two soft sets over } X \text { defined as follows: }
\end{aligned}
$$$$
G\left(e_{1}\right)=\left\{h_{1}, h_{3}\right\}, \quad G\left(e_{2}\right)=\left\{h_{2}, h_{4}\right\}, H\left(e_{1}\right)=\left\{h_{2}\right\}, \quad H\left(e_{2}\right)=\left\{h_{2}\right\} .
$$$$
d_{s w}(G, E)=(A, E) \text {, where: }
$$$$
A\left(e_{1}\right)=\left\{h_{1}, h_{3}\right\}, \quad A\left(e_{2}\right)=\left\{h_{2}, h_{4}\right\} \text {, and } d_{s w}(H, E)=(B, E) \text {, where: }
$$$$
B\left(e_{1}\right)=\left\{h_{1}\right\}, \quad B\left(e_{2}\right)=\left\{h_{1}, h_{2}\right\} .
$$$$
\text { so } d_{s w}(G, E) \tilde{\cup} d_{s w}(H, E)=(C, E) \text {, where: }
$$$$
C\left(e_{1}\right)=\left\{h_{1}, h_{3}\right\}, \quad C\left(e_{2}\right)=\left\{h_{2}, h_{4}\right\} \text {. Also, }
$$$$
d_{s w}((G, E) \tilde{\cup}(H, E))=(D, E) \text {, where: }
$$$$
D\left(e_{1}\right)=\left\{h_{1}, h_{3}, h_{4}\right\}, \quad D\left(e_{2}\right)=X \text {. }
$$$$
\text { Hence, } d_{s w}\left(\left(G_{E}\right) \tilde{\cup}\left(H_{E}\right)\right) \tilde{\Phi} d_{s w}\left(G_{E}\right) \tilde{\cup} d_{s w}\left(H_{E}\right) \text {. }
$$

Theorem 3.5. Let $\mathcal{S W}$ be a soft weak structure over $X$ with a fixed set of parameters $E$ and $(F, E),(G, E) \in S S(X)_{E}$. Then:

$$
\begin{aligned}
& \text { 1. } i_{s w}(F, E) \tilde{\cup} i_{s w}(G, E) \tilde{\subseteq} i_{s w}((F, E) \tilde{\cup}(G, E)) . \\
& \text { 2. } c_{s w}((F, E) \tilde{\cap}(G, E)) \tilde{\subseteq} c_{s w}(F, E) \tilde{\cap} c_{s w}(G, E) \text {. } \\
& \text { 3. } d_{s w}((F, E) \tilde{\cap}(G, E)) \tilde{\subseteq} d_{s w}(F, E) \tilde{\cap} d_{s w}(G, E) \text {. }
\end{aligned}
$$

Proof. 1. Since $i_{s w}(F, E) \tilde{\subseteq}(F, E)$ and $i_{s w}(G, E) \tilde{\subseteq}(G, E)$ from Theorem 2.1 (1), $i_{s w}(F, E) \tilde{\cup} i_{s w}(G, E) \tilde{\subseteq}((F, E) \tilde{\cup}(G, E))$. But, $i_{s w}((F, E) \tilde{\cup}(G, E))$ is the largest $\mathcal{S} \mathcal{W}$-open soft set that contained in $(F, E) \tilde{\cup}(G, E)$. Hence, $i_{s w}(F, E) \tilde{\cup} i_{s w}(G, E) \tilde{\subseteq} i_{s w}((F, E) \tilde{\cup}(G, E))$.

2. Follows from Theorem 2.1 (3).

3. Follows from Theorem 3.3 (1).

Remark 3.3. Let $\mathcal{S W}$ be a soft weak structure over $X$ with a fixed set of parameters $E$. Then, the following properties are not true in general:

$$
\text { 1. } i_{s w}(\tilde{X})=\tilde{X} \text {. }
$$


2. $c_{s w}(\tilde{\varphi})=\tilde{\varphi}$.

The following example supports our claim.

Example 3.2. $\quad$ Refer to Example 3.1, $i_{s w}(\tilde{X})=(A, E) \neq \tilde{X}$, where:

$A\left(e_{1}\right)=\left\{h_{1}, h_{2}\right\}, \quad A\left(e_{2}\right)=\left\{h_{2}, h_{3}, h_{4}\right\}$.

Also, $c_{s w}(\tilde{\varphi})=(B, E) \neq \tilde{\varphi}$, where:

$B\left(e_{1}\right)=\left\{h_{3}, h_{4}\right\}, \quad A\left(e_{2}\right)=\{h 1\}$.

Theorem 3.6. Let $\mathcal{S W}$ be a soft weak structure over $X$ with a fixed set of parameters $E$ and $(F, E) \in S S(X)_{E}$. Then:
1. $i_{s w}\left(c_{s w}\left(i_{s w}\left(c_{s w}(F, E)\right)\right)\right)=i_{s w}\left(c_{s w}(F, E)\right)$.
2. $c_{s w}\left(i_{s w}\left(c_{s w}\left(i_{s w}(F, E)\right)\right)\right)=c_{s w}\left(i_{s w}(F, E)\right)$.

Proof. 1. Let $(F, E) \in S S(X)_{E}$. We have $i_{s w}\left(c_{s w}(F, E)\right) \tilde{\subseteq} c_{s w}(F, E)$. By Theorem $2.1(3), c_{s w}\left(i_{s w}\left(c_{s w}(F, E)\right)\right) \tilde{\simeq} c_{s w}(F, E)$ and hence $i_{s w}\left(c_{s w}\left(i_{s w}\left(c_{s w}(F, E)\right)\right)\right) \tilde{\simeq} i_{s w}\left(c_{s w}(F, E)\right)$. On the other hand, $i_{s w}\left(c_{s w}(F, E)\right) \tilde{\widetilde{C}} c_{s w}\left(i_{s w}\left(c_{s w}(F, E)\right)\right)$. It follows that $i_{s w}\left(c_{s w}(F, E)\right) \underline{\tilde{\subseteq}} i_{s w}\left(c_{s w}\left(i_{s w}\left(c_{s w}(F, E)\right)\right)\right)$. This completes the proof.

2. By a similar way.

4. The Soft Structures $\pi(s w o), \sigma(s w o), \alpha(s w o), \beta(s w o)$ and $\rho(s w o)$

In this section, we introduce and study properties of the soft structures $\pi(s w o)$, $\sigma(s w o), \alpha(s w o), \beta(s w o)$ and $\rho(s w o)$ in detail.

Definition 4.1. Let $\mathcal{S W}$ be a soft weak structure over $X$ with a fixed set of parameters $E$ and $(F, E) \in S S(X)_{E}$. Then:

1. $(F, E) \in \pi(s w o)$ if and only if $(F, E) \tilde{\subseteq} i_{s w}\left(c_{s w}(F, E)\right)$ and $(F, E) \in \pi(s w c)$ if and only if $c_{s w}\left(i_{s w}(F, E)\right) \widetilde{\simeq}(F, E)$.

2. $(F, E) \in \sigma(s w o)$ if and only if $(F, E) \tilde{\subseteq} c_{s w}\left(i_{s w}(F, E)\right)$ and $(F, E) \in \sigma(s w c)$ if and only if $i_{s w}\left(c_{s w}(F, E)\right) \tilde{\simeq}(F, E)$.

3. $(F, E) \in \alpha(s w o)$ if and only if $(F, E) \tilde{\subseteq} i_{s w}\left(c_{s w}\left(i_{s w}(F, E)\right)\right)$ and $(F, E) \in$ $\alpha(s w c)$ if and only if $c_{s w}\left(i_{s w}\left(c_{s w}(F, E)\right)\right) \tilde{\subseteq}(F, E)$.

4. $(F, E) \in \beta(s w o)$ if and only if $(F, E) \underset{\widetilde{\tilde{c}}}{\tilde{\tilde{c}}} c_{s w}\left(i_{s w}\left(c_{s w}(F, E)\right)\right)$ and $(F, E) \in$ $\beta(s w c)$ if and only if $i_{s w}\left(c_{s w}\left(i_{s w}(F, E)\right)\right) \underline{\widetilde{\tilde{C}}}(F, E)$.

5. $(F, E) \in \rho(s w o)$ if and only if $(F, E) \tilde{\tilde{C}} i_{s w}\left(c_{s w}(F, E)\right) \tilde{\cup} c_{s w}\left(i_{s w}(F, E)\right)$ and $(F, E) \in \rho(s w c)$ if and only if $i_{s w}\left(c_{s w}(F, E)\right) \tilde{\cap} c_{s w}\left(i_{s w}(F, E)\right) \tilde{\subseteq}(F, E)$. 
Theorem 4.1. Let $\mathcal{S W}$ be a soft weak structure over $X$ with a fixed set of parameters $E$ and $(F, E) \in S S(X)_{E}$. Then:

1. Arbitrary soft union of any members of $\pi($ swo) [resp. $\sigma(s w o), \alpha(s w o), \beta(s w o)$ and $\rho(s w o)]$ is $\pi(s w o)$ [resp. $\sigma(s w o), \alpha(s w o), \beta(s w o)$ and $\rho(s w o)]$.

2. Arbitrary soft intersection of any members of $\pi(s w c)$ [resp. $\sigma(s w c), \alpha(s w c)$, $\beta(s w c)$ and $\rho(s w c)]$ is $\pi(s w c)$ [resp. $\sigma(s w c), \alpha(s w c), \beta(s w c)$ and $\rho(s w c)]$.

Proof. 1. We will give the proof for the case of $\pi(s w o)$, the other cases are similar. Let $\left\{\left(F_{j}, E\right): j \in J\right\} \subseteq \pi(s w o)$. Then, $\forall j \in J,\left(F_{j}, E\right) \tilde{\subseteq} i_{s w}\left(c_{s w}\left(\left(F_{j}, E\right)\right)\right)$. It follows that $\tilde{U}_{j}\left(F_{j}, E\right) \tilde{\subseteq} \tilde{U}_{j} i_{s w}\left(c_{s w}\left(\left(F_{j}, E\right)\right)\right) \tilde{\subseteq} i_{s w}\left(\tilde{\bigcup}_{j} c_{s w}\left(\left(F_{j}, E\right)\right)\right) \tilde{\subseteq} i_{s w}\left(c_{s w}\left(\tilde{U}_{j}\left(F_{j}, E\right)\right)\right)$ from Theorems 3.4 (2) and 3.5 (1). Hence, $\tilde{U}_{j}\left(F_{j}, E\right) \in \pi(s w o) \forall j \in J$. The rest of the proof is similar.

2. Obvious from (1).

Is the family $\pi(s w o)$ [resp. $\sigma(s w o), \alpha(s w o), \beta(s w o)$ and $\rho(s w o)]$ forms a (supra) soft topology? The answer is no for the next remark.

Remark 4.1. The finite soft intersection of two $\pi(s w o)$ [resp. $\sigma(s w o), \alpha(s w o)$, $\beta(s w o)$ and $\rho(s w o)]$ soft sets needs not to be $\pi($ swo $)$ [resp. $\sigma(s w o), \alpha(s w o), \beta(s w o)$ and $\rho($ swo $)]$, as shown in the following examples.

Examples 4.1. 1. Suppose that there are three jobs in the universe $\mathrm{X}$ given by $X=\left\{j_{1}, j_{2}, j_{3}\right\}$. Let $E=\left\{e_{1}, e_{2}\right\}$ be the set of decision parameters which stand for "salary" and "position" respectively.

Let $\left(F_{1}, E\right),\left(F_{2}, E\right),\left(F_{3}, E\right)$ be three soft sets over the common universe X, which describe the flexibility of the jobs, where

$F_{1}\left(e_{1}\right)=\left\{j_{1}\right\}, \quad F_{1}\left(e_{2}\right)=\left\{j_{1}\right\}$,

$F_{2}\left(e_{1}\right)=\left\{j_{1}, j_{2}\right\}, \quad F_{2}\left(e_{2}\right)=\left\{j_{1}, j_{2}\right\}$,

$F_{3}\left(e_{1}\right)=\left\{j_{2}, j_{3}\right\}, \quad F_{3}\left(e_{2}\right)=\left\{j_{2}, j_{3}\right\}$.

Then, $\mathcal{S W}=\left\{\tilde{\varphi},\left(F_{1}, E\right),\left(F_{2}, E\right),\left(F_{3}, E\right)\right\}$ defines a soft weak structure on $X$.

Hence, the sets $(G, E)$ and $(H, E)$ which defined as follows:

$G\left(e_{1}\right)=\left\{j_{1}, j_{3}\right\}, \quad G\left(e_{2}\right)=\left\{j_{1}, j_{3}\right\}$,

$H\left(e_{1}\right)=\left\{j_{2}, j_{3}\right\}, \quad H\left(e_{2}\right)=\left\{j_{2}, j_{3}\right\}$,

are $\pi(s w)($ resp. $\beta(s w))$-open soft sets, but their soft intersection $(G, E) \tilde{\cap}(H, E)=$

$(M, E)$ is not $\pi(s w)$ (resp. $\beta(s w))$-open soft set, where:

$M\left(e_{1}\right)=\left\{j_{3}\right\}, \quad M\left(e_{2}\right)=\left\{j_{3}\right\}$.

2. Let $X=\left\{j_{1}, j_{2}, j_{3}\right\}, E=\left\{e_{1}, e_{2}\right\}$ and $\mathcal{S W}=\left\{\tilde{\varphi},\left(F_{1}, E\right),\left(F_{2}, E\right)\right\}$, where:

$\left(F_{1}, E\right),\left(F_{2}, E\right)$ are soft sets over $X$ defined as follows:

$F_{1}\left(e_{1}\right)=\left\{j_{1}, j_{2}\right\}, \quad F_{1}\left(e_{2}\right)=\left\{j_{1}\right\}$, 
$F_{2}\left(e_{1}\right)=\left\{j_{1}, j_{3}\right\}, \quad F_{2}\left(e_{2}\right)=\left\{j_{2}, j_{3}\right\}$.

Hence, the sets $\left(F_{1}, E\right),\left(F_{2}, E\right)$ are $\alpha(s w)$-open soft sets, but their soft intersection $\left(F_{1}, E\right) \tilde{\cap}\left(F_{2}, E\right)=(L, E)$ is not $\alpha(s w)$-open soft, where:

$L\left(e_{1}\right)=\left\{j_{1}\right\}, \quad L\left(e_{2}\right)=\varphi$.

3. In (2), the soft sets $(G, E)$ and $(H, E)$ are $\sigma(s w)$-open soft sets, which defined as follows:

$G\left(e_{1}\right)=\left\{j_{1}, j_{2}\right\}, \quad G\left(e_{2}\right)=\left\{j_{1}, j_{3}\right\}$,

$H\left(e_{1}\right)=\left\{j_{1}, j_{3}\right\}, \quad H\left(e_{2}\right)=\left\{j_{2}, j_{3}\right\}$.

But, their soft intersection $(G, E) \tilde{\cap}(H, E)=(N, E)$ where $N\left(e_{1}\right)=\left\{j_{1}\right\}$,

$N\left(e_{2}\right)=\left\{j_{3}\right\}$, is not $\sigma(s w)$-open soft.

4. Suppose that there are three phones in the universe $\mathrm{X}$ given by $X=\left\{p_{1}, p_{2}, p_{3}\right\}$. Let $E=\left\{e_{1}, e_{2}\right\}$ be the set of decision parameters which stand for "expensive" and "beautiful" respectively.

Let $\left(F_{1}, E\right),\left(F_{2}, E\right),\left(F_{3}, E\right)$ be soft set over the common universe $\mathrm{X}$, which describe the composition of the phones, where

$F_{1}\left(e_{1}\right)=\left\{p_{1}\right\}, \quad F_{1}\left(e_{2}\right)=\left\{p_{1}\right\}$,

$F_{2}\left(e_{1}\right)=\left\{p_{1}, p_{2}\right\}, \quad F_{2}\left(e_{2}\right)=\left\{p_{1}, p_{2}\right\}$,

$F_{3}\left(e_{1}\right)=\left\{p_{2}, p_{3}\right\}, \quad F_{3}\left(e_{2}\right)=\left\{p_{2}, p_{3}\right\}$.

Then, $\mathcal{S W}=\left\{\tilde{\varphi},\left(F_{1}, E\right),\left(F_{2}, E\right),\left(F_{3}, E\right)\right\}$ defines a soft weak structure on $X$. Hence, the soft sets $(G, E)$ and $(H, E)$ which defined as follows:

$G\left(e_{1}\right)=\left\{p_{1}, p_{3}\right\}, \quad G\left(e_{2}\right)=\left\{p_{1}, p_{3}\right\}$,

$H\left(e_{1}\right)=\left\{p_{2}, p_{3}\right\}, \quad H\left(e_{2}\right)=\left\{p_{2}, p_{3}\right\}$,

are $\rho(s w)$-open soft sets, but their soft intersection $(G, E) \tilde{\cap}(H, E)=(M, E)$, where $M\left(e_{1}\right)=\left\{p_{3}\right\}, \quad M\left(e_{2}\right)=\left\{p_{3}\right\}$, is not $\rho(s w)$-open soft set.

Based on Theorem 4.1 and Remark 4.1, we have the following theorem.

Theorem 4.2. For any soft weak structure $\mathcal{S W}$, each of the soft structures $\pi($ swo $)$ [resp. $\sigma(s w o), \alpha(s w o), \beta(s w o)$ and $\rho(s w o)]$ is soft generalized topology [10].

Theorem 4.3. For any soft weak structure $\mathcal{S W}$ on $X$, we have swo $(X) \subseteq \alpha($ swo $) \subseteq$ $\sigma(s w o) \subseteq \rho(s w o) \subseteq \beta($ swo $)$ and $\alpha(s w o) \subseteq \pi(s w o) \subseteq \rho(s w o)$.

Proof. Let $(F, E) \in \operatorname{swo}(X)$, then $i_{s w}(F, E)=(F, E)$ from Theorem 3.1 (1). Then, $i_{s w}(F, E)=(F, E) \tilde{\subseteq} c_{s w}\left(i_{s w}(F, E)\right)$ from Theorem $2.1(1)$. It follows, $(F, E) \tilde{\subseteq} i_{s w}\left(c_{s w}\right.$ $\left.\left(i_{s w}(F, E)\right)\right)$ from Theorem $2.1(3)$. Thus, $(F, E) \in \alpha(s w o)$.

Also, if $(F, E) \in \alpha(s w o)$, then $(F, E) \tilde{\tilde{C}} i_{s w}\left(c_{s w}\left(i_{s w}(F, E)\right)\right) \tilde{\tilde{C}} c_{s w}\left(i_{s w}(F, E)\right)$, hence $\alpha(s w o) \subseteq \sigma(s w o)$.

It is clear that $\sigma(s w o) \subseteq \rho(s w o)$ from Definition 4.1. Furthermore,

$i_{s w}\left(c_{s w}(F, E)\right) \tilde{\subseteq} c_{s w}\left(i_{s w}\left(c_{s w}(F, E)\right)\right)$ and $c_{s w}\left(i_{s w}(F, E)\right) \tilde{\subseteq} c_{s w}\left(i_{s w}\left(c_{s w}(F, E)\right)\right)$ from Theorem 2.1. Thus, $\left.(F, E) \tilde{\subseteq}\left[i_{s w}\left(c_{s w}(F, E)\right)\right] \tilde{\cup}\left[c_{s w}\left(i_{s w}(F, E)\right)\right]\right] \tilde{\subseteq} c_{s w}\left(i_{s w}\left(c_{s w}(F, E)\right)\right)$ and so $\rho(s w o) \subseteq \beta($ swo $)$. 
Now, if $(F, E) \in \alpha(s w o)$, then $(F, E) \tilde{\subseteq} i_{s w}\left(c_{s w}\left(i_{s w}(F, E)\right)\right) \tilde{\subseteq} i_{s w}\left(c_{s w}(F, E)\right)$ by Theorem 2.1 (3) so that $\alpha(s w o) \subseteq \pi(s w o)$. Also, $\pi(s w o) \subseteq \rho(s w o)$ follows directly from Definition 4.1.

Definition 4.2. Let $\mathcal{S W}$ be a soft weak structure over $X$ with a fixed set of parameters $E$ and $(F, E) \in S S(X)_{E}$. Then, $(F, E)$ is called $\mathcal{S W}$-clopen soft set if it is both $\mathcal{S W}$-open soft and $\mathcal{S W}$-closed soft set.

Theorem 4.4. Let $\mathcal{S W}$ be any soft weak structure on $X$ and $(F, E) \in S S(X)_{E}$. If $(F, E)$ is $\mathcal{S} \mathcal{W}$-clopen soft set, then $(F, E) \in \alpha($ swo $)$ and $(F, E)^{\tilde{c}} \in \pi($ swo $)$.

Proof. Let $(F, E)$ be a $\mathcal{S W}$-clopen soft set on $\mathcal{S W}$. Then, $i_{s w}(F, E)=(F, E)=$ $c_{s w}(F, E)$ from Theorem 3.1. Also, we have $(F, E)=i_{s w}\left(i_{s w}(F, E)\right) \tilde{\subseteq} i_{s w}\left(c_{s w}\left(i_{s w}(F, E)\right)\right)$. Hence, $(F, E) \in \alpha(s w o)$. On the other hand, $(F, E)^{\tilde{c}}=i_{s w}(F, E)^{\tilde{c}}=i_{s w}\left(i_{s w}(F, E)^{\tilde{c}}\right) \tilde{\subseteq} i_{s w}\left(c_{s w}(F, E)^{\tilde{c}}\right)$. Thus, $(F, E)^{\tilde{c}} \in \pi(s w o)$.

Remark 4.2. The converse of Theorem 4.4 is not true in general as shown in the following example.

Example 4.1. Suppose that there are four alternatives in the universe of cars $X=$ $\left\{c_{1}, c_{2}, c_{3}, c_{4}\right\}$ and consider $E=\left\{e_{1}, e_{2}\right\}$ be the set of decision parameters which stand for "Motor" and "color" respectively. Let $\mathcal{S W}=\left\{\tilde{\varphi},\left(F_{1}, E\right),\left(F_{2}, E\right),\left(F_{3}, E\right),\left(F_{4}, E\right)\right\}$, where $\left(F_{1}, E\right),\left(F_{2}, E\right),\left(F_{3}, E\right),\left(F_{4}, E\right)$ are four soft sets over $X$ representing the attractiveness of the cars which Mr. $A$ and Mr. $B$ are going to buy defined as follows:

$F_{1}\left(e_{1}\right)=\left\{c_{4}\right\}, \quad F_{1}\left(e_{2}\right)=\left\{c_{4}\right\}$

$F_{2}\left(e_{1}\right)=\left\{c_{1}, c_{2}\right\}, \quad F_{2}\left(e_{2}\right)=\left\{c_{2}, c_{3}\right\}$,

$F_{3}\left(e_{1}\right)=\left\{c_{2}, c_{3}\right\}, \quad F_{3}\left(e_{2}\right)=\left\{c_{1}, c_{2}\right\}$,

$F_{4}\left(e_{1}\right)=\left\{c_{1}, c_{2}, c_{4}\right\}, \quad F_{4}\left(e_{2}\right)=\left\{c_{4}\right\}$.

Consider the soft set $(G, E)$, where:

$G\left(e_{1}\right)=\left\{c_{1}, c_{2}, c_{3}\right\}, \quad G\left(e_{2}\right)=\left\{c_{1}, c_{2}, c_{3}\right\}$,

we have $(G, E) \in \alpha(s w o)$ and $(G, E)^{\tilde{c}} \in \pi(s w o)$. But, $(G, E)$ is not $\mathcal{S} \mathcal{W}$-clopen soft set.

Theorem 4.5. Let $\mathcal{S W}$ be any soft weak structure on $X$ and $(F, E) \in S S(X)_{E}$. If there exists a $\mathcal{S W}$-open soft set $(G, E)$ such that $(G, E) \tilde{\subseteq}(F, E) \tilde{\subseteq} c_{s w}(G, E)$, then $(F, E) \in \sigma(s w o)$.

Proof. Let $(G, E) \tilde{\subseteq}(F, E) \tilde{\simeq} c_{s w}(G, E)$ where $(G, E) \in \operatorname{swo}(X)$. Then, $(G, E)=$ $i_{s w}(G, E) \tilde{\simeq} i_{s w}$

$(F, E)$, and hence $(F, E) \tilde{\tilde{\subseteq}} c_{s w}(G, E) \subseteq \tilde{\subseteq} c_{s w}\left(i_{s w}(F, E)\right)$. Thus, $(F, E) \in \sigma(s w o)$.

Theorem 4.6. Let $\mathcal{S W}$ be any soft weak structure on $X$ and $(F, E) \in \beta($ swo $)$. Then, $(F, E)=(A, E) \tilde{\cap}(B, E)$ for some $(A, E) \in \sigma($ swo $)$ and $(B, E)$ is $\mathcal{S W}$-soft dense set.

Proof. Let $(F, E) \in \beta(s w o)$. Then, $(F, E) \tilde{\tilde{\subseteq}} c_{s w}\left(i_{s w}\left(c_{s w}(F, E)\right)\right)$ and so $c_{s w}(F, E) \tilde{\tilde{\tilde{c}}} c_{s w}$ $\left(i_{s w}\left(c_{s w}(F, E)\right)\right)$. Furthermore, $c_{s w}\left(i_{s w}\left(c_{s w}(F, E)\right)\right) \tilde{\subseteq} c_{s w}(F, E)$. Thus, 
$c_{s w}\left(i_{s w}\left(c_{s w}(F, E)\right)\right)=c_{s w}(F, E)$. Take $c_{s w}(F, E)=(A, E)$, then $c_{s w}\left(i_{s w}(A, E)\right)=$ $(A, E)$. Hence, $(A, E) \in \sigma(s w o)$. On the other hand, if we take $(B, E)=(F, E) \tilde{\cup}(A, E)^{\tilde{c}}$, then $(B, E)=(F, E) \tilde{\cup}\left(c_{s w}(F, E)\right)^{\tilde{c}}$. Hence,

$c_{s w}(B, E)=c_{s w}\left[(F, E) \tilde{\cup}\left(c_{s w}(F, E)\right)^{\tilde{c}}\right] \tilde{\supseteq} c_{s w}(F, E) \tilde{\cup}\left(c_{s w}(F, E)\right)^{\tilde{c}}=\tilde{X}$. Thus, $(B, E)$ is $\mathcal{S W}$-soft dense set and $(F, E)=(A, E) \tilde{\cap}(B, E)$.

Remark 4.3. The converse of Theorem 4.6 is not true in general as shown in the following example.

Example 4.2. Suppose that there are four alternatives in the universe of watches $X=$ $\left\{w_{1}, w_{2}, w_{3}, w_{4}\right\}$ and consider $E=\left\{e_{1}, e_{2}\right\}$ be the set of decision parameters which stand for "expensive" and "originality" respectively. Let $\mathcal{S W}=\left\{\tilde{\varphi},\left(F_{1}, E\right),\left(F_{2}, E\right)\right.$,

$\left.\left(F_{3}, E\right),\left(F_{4}, E\right)\right\}$, where $\left(F_{1}, E\right),\left(F_{2}, E\right),\left(F_{3}, E\right),\left(F_{4}, E\right)$ are four soft sets over $X$ representing the quality of the watches which Mr. $M$ and Mr. $N$ are going to buy defined as follows:

$F_{1}\left(e_{1}\right)=\left\{w_{1}, w_{2}\right\}, \quad F_{1}\left(e_{2}\right)=\left\{w_{1}, w_{3}\right\}$

$F_{2}\left(e_{1}\right)=\left\{w_{2}, w_{3}\right\}, \quad F_{2}\left(e_{2}\right)=\left\{w_{2}, w_{3}, w_{4}\right\}$

$F_{3}\left(e_{1}\right)=\left\{w_{1}\right\}, \quad F_{3}\left(e_{2}\right)=\left\{w_{1}\right\}$

$F_{4}\left(e_{1}\right)=\left\{w_{2}, w_{4}\right\}, \quad F_{4}\left(e_{2}\right)=\left\{w_{1}, w_{2}, w_{4}\right\}$.

Consider the soft sets $(J, E)$ and $(K, E)$, where:

$J\left(e_{1}\right)=\left\{w_{2}, w_{3}\right\}, \quad J\left(e_{2}\right)=\left\{w_{2}, w_{3}, w_{4}\right\}$ and

$K\left(e_{1}\right)=\left\{w_{1}, w_{3}, w_{4}\right\}, \quad K\left(e_{2}\right)=\left\{w_{1}, w_{3}\right\}$.

Thus, $(J, E) \in \sigma(s w o)$ and $(K, E)$ is $\mathcal{S} \mathcal{W}$-soft dense set. But, $(J, E) \tilde{\cap}(K, E)=(L, E) \notin$ $\beta(s w o)$, where:

$L\left(e_{1}\right)=\left\{w_{3}\right\}, \quad L\left(e_{2}\right)=\left\{w_{3}\right\}$.

Theorem 4.7. Let $\mathcal{S W}$ be any soft weak structure on $X$ and $(F, E) \in S S(X)_{E}$. If there exists an $\beta\left(\right.$ sw)-open soft set $(G, E)$ such that $(G, E) \tilde{\subseteq}(F, E) \tilde{\subseteq} c_{s w}(G, E)$, then $(F, E) \in \beta($ swo $)$.

Proof. Let $(G, E) \tilde{\subseteq}(F, E) \tilde{\subseteq} c_{s w}(G, E)$ where $(G, E) \in \beta(s w o)$. Then, $(G, E) \tilde{\subseteq} c_{s w}\left(i_{s w}\left(c_{s w}(G, E)\right)\right)$. Hence,

$(F, E) \underline{\tilde{\subseteq}} c_{s w}(G, E) \tilde{\subseteq} c_{s w}\left(i_{s w}\left(c_{s w}(G, E)\right)\right) \tilde{\tilde{\subseteq}} c_{s w}\left(i_{s w}\left(c_{s w}(F, E)\right)\right)$ from Theorem 2.1 and so $(F, E) \in \beta(s w o)$.

\section{Conclusion}

In this paper, firstly, we have listed the main properties which give the deviation between soft weak structure [17] and that in soft topological spaces [15] and supra soft topological spaces [7]. Secondly, we introduced the soft structures $\pi(s w o)$, $\sigma(s w o), \alpha(s w o), \beta(s w o)$ and $\rho(s w o)$. Also, we studied their basic properties. In future, the generalization of these concepts by using the soft ideal notions [12] will be introduced and the future research will be undertaken in this direction. Also, the soft structure $r(s w o)$ will be studied. We predict that many of these parameters studied and the new concepts introduced in this paper have, in fact, applications in real world situations as shown in [1], and therefore I believe that this is an extra justification for the work conducted in this paper. 


\section{Compliance with Ethical Standards}

This study is funded by Northern Borders University, Rafha, KSA. Also, I declare that, I have no conflict of interest. Furthermore, this article does not contain any studies with human participants (resp. any studies with animals) performed by the author.

\section{Acknowledgements}

The author expresses his sincere thanks to the reviewers for their valuable suggestions. The author is also thankful to the editors-in-chief and managing editors for their important comments which helped to improve the presentation of the paper.

\section{R E F E R E N C E S}

1. S. ARPAD, Minimal structures, generalized topologies, and ascending systems should not be studied without generalized uniformities, Faculty of Sciences and Mathematics University of Nis, 21 (1) (2007), 87-97

2. Á. CsÁsÁzr, Generalized open sets, Acta Math. Hungar., 75 (1997), 65-87.

3. Á. CsÁsÁzR, Generalized topology, generalized continuity, Acta Math. Hungar., 96 (2002), 351-357.

4. Á. CsÁsÁzR, Generalized open sets in generalized topologies, Acta Math. Hungar., 106 (2005), 53-66.

5. Á. CsÁsÁzR, Weak structures, Acta Math. Hungar., 131 (2011), 193-195.

6. E. Ekici, On weak structures due to Csásázr, Acta Math. Hungar., 134 (2012), 565-570.

7. S. A. El-Sheikh and A. M. Abd El-Latif, Decompositions of some types of supra soft sets and soft continuity, International Journal of Mathematics Trends and Technology, 9 (1) (2014), 37-56.

8. A. Ghareeb and M. M. Khalaf, On weak structures, Fasc. Matematica, Tom XXII (2015), Issue No. 1, 129-134.

9. R. Gowri And S.Vembu, Soft minimal and soft biminimal spaces, International J. of Mathematical Sciences \& Applications, 5 (2) (2015), 447-455.

10. T. Jyотнis And J. J. Sunil, On soft generalized topological spaces, Journal of New Results in Science, 4 (2014), 1-15.

11. A. Kandil, O. A. E. Tantawy, S. A. El-Sheikh and A. M. Abd El-Latif, $\gamma-$ operation and decompositions of some forms of soft continuity in soft topological spaces, Ann. Fuzzy Math. Inform., 7 (2) (2014), 181-196.

12. A. Kandil, O. A. E. Tantawy, S. A. El-Sheikh and A. M. Abd El-latif, Soft ideal theory, Soft local function and generated soft topological spaces, Appl. Math. Inf. Sci., 8 (4) (2014), 1595-1603.

13. H. Maki, J. Umehara And T. NoIRI, Every topological space is pre- $T$, Mem. Fac. Sci. Kochi. Univ. Ser. A Math., 17 (1996), 33-42. 
14. D. A. Molodtsov, Soft set theory-firs tresults, Comput. Math. Appl., 37 (1999), 19-31.

15. M. Shabir And M. NAz, On soft topological spaces, Comput. Math. Appl., 61 (2011), 1786-1799.

16. A. M. Zahran, A. K. Musa and A. Ghareeb, Generalized closed sets and some separation axioms on weak structure, Hacettepe Journal of Mathematics and Statistics, 44 (3) (2015), 669-677.

17. A. H. Zakari, A. Ghareeb and Saleh Omran, On soft weak structures, Soft Computing, Published online: 4 April(2016), DOI 10.1007/s00500-016-2136-8, 1-

18. I. Zorlutuna, M. Akdag, W.K. Min and S. Atmaca, Remarks on soft topological spaces, Ann. Fuzzy Math. Inform., 3 (2) (2012), 171-185.

\author{
A. M. Abd El-latif \\ Northern Borders University \\ Faculty of Arts and Science \\ Department of Mathematics \\ Rafha, KSA. \\ Ain Shams University \\ Faculty of Education \\ Mathematics Department \\ Roxy, 11341, Cairo, Egypt \\ alaa_8560@yahoo.com, Alaa.ali@nbu.edu.sa
}

